\title{
SurvivinT34A increases the therapeutic efficacy of arsenic trioxide in mouse hepatocellular carcinoma models
}

\author{
ANLIANG HUANG ${ }^{1,2^{*}}$, DAN YUE ${ }^{1 *}$, DANYING LIAO ${ }^{3}$, LIULIU CHENG $^{1}$, \\ JINHU MA ${ }^{1}$, YUQUAN WEI ${ }^{1}$, AIPING TONG ${ }^{1}$ and PING CHENG ${ }^{1}$ \\ ${ }^{1}$ State Key Laboratory of Biotherapy/Collaborative Innovation Center for Biotherapy/Cancer Center, \\ West China Hospital; ${ }^{2}$ Department of Pathology, West China Second Hospital; ${ }^{3}$ Department of Pathology, \\ West China Hospital, Sichuan University, Chengdu, Sichuan 610041, P.R. China
}

Received March 1, 2016; Accepted June 14, 2016

DOI: $10.3892 / o r .2016 .5161$

\begin{abstract}
Arsenic trioxide (ATO) has demonstrated clinical efficacy in acute promyelocytic leukemia (APL) and in vitro activity in various solid tumors. $\mathrm{As}_{2} \mathrm{O}_{3}$ as single agent exhibits poor efficacy for treatment of hepatocellular carcinoma (HCC) in phase II trial, suggesting that new modalities of treatment with enhanced therapeutic effect and alleviated toxicity are needed for application of $\mathrm{As}_{2} \mathrm{O}_{3}$ on patients with HCC. Survivin is the strongest inhibitor of apoptosis protein overexpressed in tumors, which has been proposed as an attractive target for new anticancer interventions. Disruption of survivin by the plasmid encoding the phosphorylation-defective mouse survivin threonine $34 \rightarrow$ alanine mutant (Msurvivin T34A plasmid) has proved a promising strategy for suppressing a variety of murine cancer. In the present study, we attempted to test Msurvivin T34A and arsenic trioxide (ATO) on a cell line and mice bearing subcutaneous tumors, with regard to their effects and mechanisms. We observed that the co-treatment with surivinT34A and ATO significantly enhanced the antitumor activity by induction of apoptosis in Hepa1-6 tumor cells in vitro, compared with control groups. The synergistic
\end{abstract}

Correspondence to: Professor Ping Cheng, State Key Laboratory of Biotherapy/Collaborative Innovation Center of Biotherapy/Cancer Center, West China Hospital, Sichuan University, 17 People's South Road, Chengdu, Sichuan 610041, P.R. China

E-mail: ping.cheng@foxmail.com

\section{*Contributed equally}

Abbreviations: ATO, arsenic trioxide; APL, acute promyelocytic leukemia; HCC, hepatocellular carcinoma; ATRA, all-trans-retinoic acid; TAL, tachypleusamebocytelysate; DOTAP, dioleyltrimethylammonium propane; ROS, reactive oxygen species; DCFH-DA, 2,7-dichlorodihydrofluorescein diacetate; NAC, N-acetyl-1-cysteine; DCF, 2,7-dichlorofluorescein; PARP, poly(ADP)-ribose polymerase

Key words: survivin, arsenic trioxide, reactive oxygen species, hepatocellular carcinoma apoptosis-inducing effect of combination of these two drugs resulted in elevation of reactive oxygen species (ROS) level which could be antagonized by the antioxidant N-acetyl-1cysteine. The combination treatment induced ROS-dependent collapse of the mitochondrial membrane potential. Moreover, the tumor growth in vivo was also remarkably inhibited by combination of surivinT34A and ATO when compared with control groups. Our findings demonstrate that the combination of surivinT34A and ATO exerted synergistic antitumor effects, providing a new perspective for clinical treatment of HCC.

\section{Introduction}

Hepatocellular carcinoma (HCC) ranks the fifth most common malignant tumor in the world. Although the outcome of HCC patients appear to be improving due to a benefit from early diagnosis and more effective treatment, the majority of HCC cases are not capable of totally surgical resection and remain insensitive to radiotherapy and chemotherapy. The overall 5-year survival rate of HCC patients has shown no improvement over the last several decades (1). Thus, more effective strategies against HCC need to be developed.

Dysregulation of apoptosis is a common feature of malignant cells and represents a significant obstacle to the treatment of human cancer. Among the regulators of apoptosis, survivin, a member of the inhibitor of apoptosis protein family, plays an important role not only in inhibiting apoptosis but also in regulating mitosis (2). Due to its function in regulation of the balance between programmed cell death and cell proliferation, survivin is vital for cancer cell survival, and have been verified to be a prognostic indicator for poor survival in several malignancies $(3,4)$. The most prominent feature of the survivin expression profile is that it is rarely detected in normal tissues, whereas it is selectively overexpressed in most tumors (5), indicating that survivin may be a rational gene-directed target for cancer therapy. Studies exploiting different strategies including antisense (6), ribozymes (7), RNAi-mediation (8), survivindirected vaccines (9), or dominant negative mutants (10) to interfere with survivin expression and function provided direct and convincing results. The study of molecular structure illustrated that survivin not only consists of homodimer, which was crucial to exerting its biological function, but also 
comprises some key functional sites, such as Cys-84, Asp-71 and Thr-34 (11,12). In particular, the Thr-34 has been identified as an important phosphorylation site by the mitotic kinase p34cdc2-cyclin B1, and the phosphorylation at Thr-34 has a direct correlation with anti-apoptotic function of survivin (13). However, Thr34 $\rightarrow$ Ala in wild-type survivin would make it a surivinT34A dominant negative mutant that lost the function being phosphorylated. This mutant is capable of interfering with the phosphorylation of endogenous survivin, reducing the proliferative potential of tumour cells and enhancing tumor cell response to apoptosis inducing anticancer agents (10).

Arsenic and its derivatives have been applied in traditional Chinese medicine for thousands of years. Arsenic trioxide (ATO) is being selected as a first and second line therapy for the treatment of both newly diagnosed and all-trans-retinoic acid (ATRA)-refractory acute promyelocytic leukemia (APL) patients (14). Besides, an overwhelming number of preclinical studies have demonstrated that ATO has the ability to induce apoptosis and inhibit tumor cell growth in a wide variety of solid tumors, including acute myeloid leukemia $(15,16)$, multiple myeloma (17), head and neck (18) and glioblastoma (19). However, preliminary reports from phase II clinical trials on patients with hepatocellular carcinoma (20), metastatic renal cell carcinoma (21), and metastatic melanoma (22) indicate that the inconstant susceptibility of various tumor cells to this drug limits its clinical application as a single chemotherapeutic agent in a wider spectrum of solid malignancies. A substantial number of preclinical studies have reported that co-treatment of ATO with other chemotherapeutic agents has a synergistic effect on tumor cells both in vitro and in xenograft mouse models (23-25). To illustrate the apoptotic activation signals and/or survival inhibition signals in combination therapy of ATO with other drugs may provide a rational molecular basis for novel chemotherapeutic strategies. Thus, we tested whether the combination therapy with mouse survivinT34A and arsenic trioxide would suppress Hepa1-6 tumor growth in a synergistic manner. Our data demonstrated that the combination therapy exhibited an enhanced antitumor activity in mouse HCC models.

\section{Materials and methods}

Reagents. Arsenic trioxide and monoclonal anti-actin were obtained from Sigma-Aldrich (St. Louis, MO, USA). Arsenic trioxide was dissolved in $1.65 \mathrm{M} \mathrm{NaOH}$ at $5 \times 10^{-2} \mathrm{M}$ as a stock solution. Monoclonal anti-caspase-9 and anti-caspase-3 were purchased from Cell Signaling Technology (Beverly, MA, USA). Polyclonal anti-PARP were obtained from Santa Cruz Biotechnology (Santa Cruz, CA, USA). The protein assay kit was purchased from Bio-Rad Laboratories (Hercules, CA, USA). In situ Cell Death Detection kit was purchased from Roche (Promega, Madison, WI, USA). All the chemicals employed in the study were of analytically pure and of culture grade.

Cell culture. The murine HCC cell line Hepa1-6 obtained from the American Type Culture Collection (ATCC; Manassas, VA, USA) was maintained in Dulbecco's modified Eagle's medium (DMEM) supplemented with $10 \%$ fetal bovine serum (FBS), $2 \mathrm{mM} \mathrm{L}$-glutamine, $100 \mathrm{U} / \mathrm{ml}$ penicillin and $100 \mu \mathrm{g} / \mathrm{ml}$ streptomycin. The cells were propagated at $37^{\circ} \mathrm{C}$ in humidified $5 \% \mathrm{CO}_{2}$ conditions. The culture medium was replaced with fresh medium every 2 days.

Plasmid and liposome preparation. The recombinant plasmids carrying PORF-9-Msurvivin T34A and PORF-9 null were purchased from Invivogen Corp. Restriction endonuclease analysis PCR and DNA sequence analysis were performed to confirm the recombinant plasmid (data not shown). The plasmid was prepared by EndoFree Plasmid Giga kit (Qiagen, Inc., Chatsworth, CA, USA). Genomic DNA, small DNA fragments, or RNA were excluded in the plasmid DNA preparation as the OD260/280 ratios were between 1.8-2.0. The DNA was eventually dissolved in sterile endotoxin free water and stored at $-20^{\circ} \mathrm{C}$ before use. The cationic lipids DOTAP (dioleyltrimethylammonium propane) and cholesterol at equimolar concentrations were dissolved in chloroform supplemented with methanol (3:1 volume ratio) in a rotary $100-\mathrm{ml}$ roundbottomed flask, then the organic solvent was rotated and removed under vacuum for $2 \mathrm{~h}$. The lipid film was hydrated in 5\% dextrose in sterile water to yield a final concentration of $2.5 \mathrm{mg} / \mathrm{ml}$. Finally, the resulting mixture was vortexed for $1 \mathrm{~min}$, and then sonicated for $10 \mathrm{~min}$ to form small unilamellar vesicles. The liposome was stored at $4^{\circ} \mathrm{C}$. The final null liposome was small multilamellar liposome in a size range of $80-100 \mathrm{~nm}$, with a zeta potential from +50 to $+60 \mathrm{mV}$. When mixed with plasmid at a weight ration of 3:1, the size of CLDC vary from 150 to $170 \mathrm{~nm}$, with zeta potential close to neutral as measured by Malvern Mastersizer.

Apoptosis analysis by flow cytometry. To investigate the apoptosis inducing effect of combination treatment, we analyzed the percentage of the early apoptotic cells by flow cytometry using the Annexin V-FITC/PI dual-labeling technique. Aliquots of $1 \times 10^{5}$ Hepa1-6 cells were plated into 6-well plates in $1 \mathrm{ml}$ culture medium. When incubated to $70 \%$ confluence, cells were treated with ATO $(2 \mu \mathrm{M})$, vector+ATO, survivinT34A, survivinT34A+ATO, or untreated control, respectively. The cells were transfected with $2 \mu \mathrm{g}$ plasmid/ $6 \mu \mathrm{g}$ liposome. Forty-eight hours after transfection, cells were harvested to be stained with Annexin V-FITC kit (Beckman Coulter, Inc., Brea, CA, USA) and to be assessed by flow cytometer.

Determination of reactive oxygen species (ROS) production. Intracellular ROS production was detected using the cellpermeable indicator 2,7-dichlorodihydrofluorescein diacetate (DCFH-DA; Sigma-Aldrich), DCFH-DA was deacetylated intracellularly by non-specific esterase, which converts into fluorescent compound 2,7-dichlorofluorescein (DCF) upon reaction with hydroxyl radical, hydrogen peroxide or peroxynitrite. Briefly, cells were harvested and resuspended in DCFH-DA dye reagent $(20 \mu \mathrm{M})$ at $1 \times 10^{6} / \mathrm{ml}$, and then were incubated at $37^{\circ} \mathrm{C}$ for $20 \mathrm{~min}$ in the black box. After dye incubation, the cells were washed and resuspended in cold PBS. The fluorescence was examined by flow cytometry.

Mitochondrial transmembrane potential measurement. Mitochondrial membrane potential $\left(\Delta \Psi_{\mathrm{m}}\right)$ was measured by flow cytometry with the mitochondrial tracking fluorescent dye Rhodamine $123(\mathrm{Ex} / \mathrm{Em}=507 \mathrm{~nm} / 529 \mathrm{~nm})$, a 
cell-permeable cationic dye, which preferentially enters mitochondria due to the highly negative potential of mitochondrial membrane. Uptake and accumulation of Rhodamine 123 in the mitochondrion are driven by $\Delta \Psi_{\mathrm{m}}$, therefore, depolarization of mitochondrial membrane potential $\left(\Delta \Psi_{\mathrm{m}}\right)$ is reflected by reduction of Rhodamine 123 staining. In brief, cells were washed twice with PBS and incubated with Rhodamine 123 (100 mg/ml; Sigma-Aldrich) at $37^{\circ} \mathrm{C}$ for $30 \mathrm{~min}$ and then monitored by flow cytometry.

Western blot analysis. Briefly, $1 \times 10^{5}$ Hepa1- 6 cells were lysed in lysis buffer. Cells were removed by scraping, and centrifuged at $12,500 \mathrm{rpm}$ for $30 \mathrm{~min}$. The protein concentration of the supernatant was determined by the Bio-Rad protein assay kit, and whole-cell lysates after denaturing were sepatated by $10 \%$ SDS-PAGE. Gels were electroblotted onto a poly(vinylidene difluoride) membrane. The membrane blots were blocked at $4^{\circ} \mathrm{C}$ in $5 \%$ non-fat dry milk overnight and incubated with each antibody at a recommended dilution for $8 \mathrm{~h}$ at $37^{\circ} \mathrm{C}$. Followed by rinsing in solution with $10 \mathrm{mM}$ Tris- $\mathrm{HCl} \mathrm{pH} \mathrm{7.5,} 100 \mathrm{mM}$ $\mathrm{NaCl}$, and $0.1 \%$ Tween-20 (TBS-T), the gels were incubated in horseradish peroxidase-conjugated secondary antibodies at a dilution of 1:10,000. The immunoreactive bands were detected by enhanced chemiluminescence (Amersham Corp., Arlington Heights, IL, USA) followed by autoradiography. Equal loading was confirmed by detection of $\beta$-actin.

Animal study. C57BL/6 female mice 6-8 weeks old, weighing 20-22 g, were obtained from Beijing WeitongLihua Biological Technology Co., Ltd. (Beijing, China). Mice were maintained in a specific pathogen-free facility with 12 -h light-dark cycles. They were observed for signs of tumor growth, activity, feeding, under the guidelines of the Institutional Animal Care and Use Committee. Hepa1-6 cell suspension at concentration of $2.5 \times 10^{6}$ cells in $100 \mu \mathrm{l}$ PBS was injected s.c. into the backs of the C57BL/6 mice. Eight days after s.c. tumor inoculation all tumors were palpable, 40 of these mice were randomly assigned into the following 5 groups $(n=8)$ : i) mice received $5 \mu \mathrm{g}$ survivinT34A/15 $\mu \mathrm{g}$ liposome complexes+ATO; ii) mice received $5 \mu \mathrm{g}$ survivinT34A/15 $\mu \mathrm{g}$ liposome complexes; iii) mice received ATO (3 mg/kg); iv) mice received vector+ATO; and v) mice received $100 \mu \mathrm{l}$ of $0.9 \% \mathrm{NaCl}$ solution (NS). They received 15 i.v. administrations via tail vein on a every 2-day basis and were monitored on a daily basis for tumor burden, belly and other abnormalities. Before each treatment, DNA solution was added to liposome solution at a ratio of $1 \mu \mathrm{g}$ DNA to $3 \mu \mathrm{g}$ liposome to form DNA/liposome complex mixture. Then, the mixture was incubated at room temperature for $30 \mathrm{~min}$. The tumors were measured in two dimensions by caliper and the mice were weighed on a every 3-day basis. Tumor volume was calculated as width ${ }^{2} \mathrm{x}$ length $\mathrm{x} 0.52$. Three days after the last administration, all mice were sacrificed and then subcutaneous tumors were extracted and weighed. Primary tumors were fixed in $4 \%$ paraformaldehyde in PBS, embedded in paraffin, and cut into 3-5 $\mu \mathrm{m}$ sections. Apoptotic index in tumor tissues were detected by Terminal deoxynucleotidyl transferase mediated dUTPnick-end labeling (TUNEL) staining according to the manufacturer's protocol (Promega). Images of the representative sections were taken using the Zeiss Axiovert 400 microscope and AxioCam MRm camera. Five equal-sized fields were randomly chosen and analyzed. Density was visualized in each field, yielding the apoptosis index.

Statistical analysis. All statistical tests were performed using the SPSS 13.0 software. Statistical comparisons concerning tumor volume, tumor weight were performed using one-way analysis of variance (ANOVA); data as means \pm SD were analyzed using unpaired Student's t-test. All P-values were two sides and P-values $<0.05$ were defined as statistical significance. Experiments were performed at least in duplicate.

\section{Results}

Effects of survivinT34A combined with arsenic trioxide on tumor cells in vitro. The apoptosis of Hepa1-6 cells in each treatment group were detected by flow cytometric analysis using Annexin V-FITC/PI staining. Both early and late apoptotic cells were represented by Annexin $\mathrm{V}$ positive and PI-negative or positive cells (right quadrants). The results revealed that treatment with survivinT34A+arsenic trioxide induced more apoptotic cells than any controls (Fig. 1). Caspases play an essential role as an executor in apoptosis pathway, their activation level directly reflects the extent of apoptosis. In intrinsic apoptosis pathway, procaspase-9 is cleaved into an active caspase, which in turn activates the effector pro-caspases, including procaspase- 3 and -7 , to execute the process of apoptosis. Active caspase- 3 results in cleavage of poly(ADP)-ribose polymerase (PARP). As shown in Fig. 2, procaspase-9, procaspase-3 and PARP were significantly cleaved by the co-treatment of survivinT $34 \mathrm{~A}+\mathrm{As}_{2} \mathrm{O}_{3}$, but cleavage of procaspase-3, -9 and PARP were hardly detected in the other 3 control treatment group, except that there was slight cleavage in single survivinT34A treatment group, which was clearly weaker than that of co-treatment group.

Role of ROS in the combined effect of survivinT34A and arsenic trioxide. It has been reported that ATO induced cell death in cancer cells by promoting the production of ROS (26). To explore the molecular mechanism for the synergistic efficacy of combinational treatment with survivinT34A+ATO, ROS production in response to survivinT34A alone and combination with ATO were examined by flow cytometry using the DHR123 fluorescence dye. As shown in Fig. 3, exposure of cells to survivinT34A alone increases cellular ROS level, but co-treatment with survivinT34A and $\mathrm{As}_{2} \mathrm{O}_{3}$ remarkably augmented this ROS level elevation. Next, we determined whether ROS elevation is crucial for the synergistic effects of survivinT34A and ATO on cell apoptosis. We found that $\mathrm{N}$-acetyl-1-cysteine, a free radical scavenger, abrogated the ROS generation enhancement of the combined treatment (Fig. 3) and significantly attenuated the efficacy of combined treatment (Fig. 4). Our results indicate that ROS elevation is necessary for the induction of apoptosis by combined treatment with survivinT34A and ATO.

SurvivinT34A combined with arsenic trioxide induces a loss of mitochondria membrane potential. Excessive ROS production may result in decrease in the mitochondrial membrane potential and subsequant acceleration of apoptosis (27). Therefore, we assessed the changes in mitochondria membrane potential 

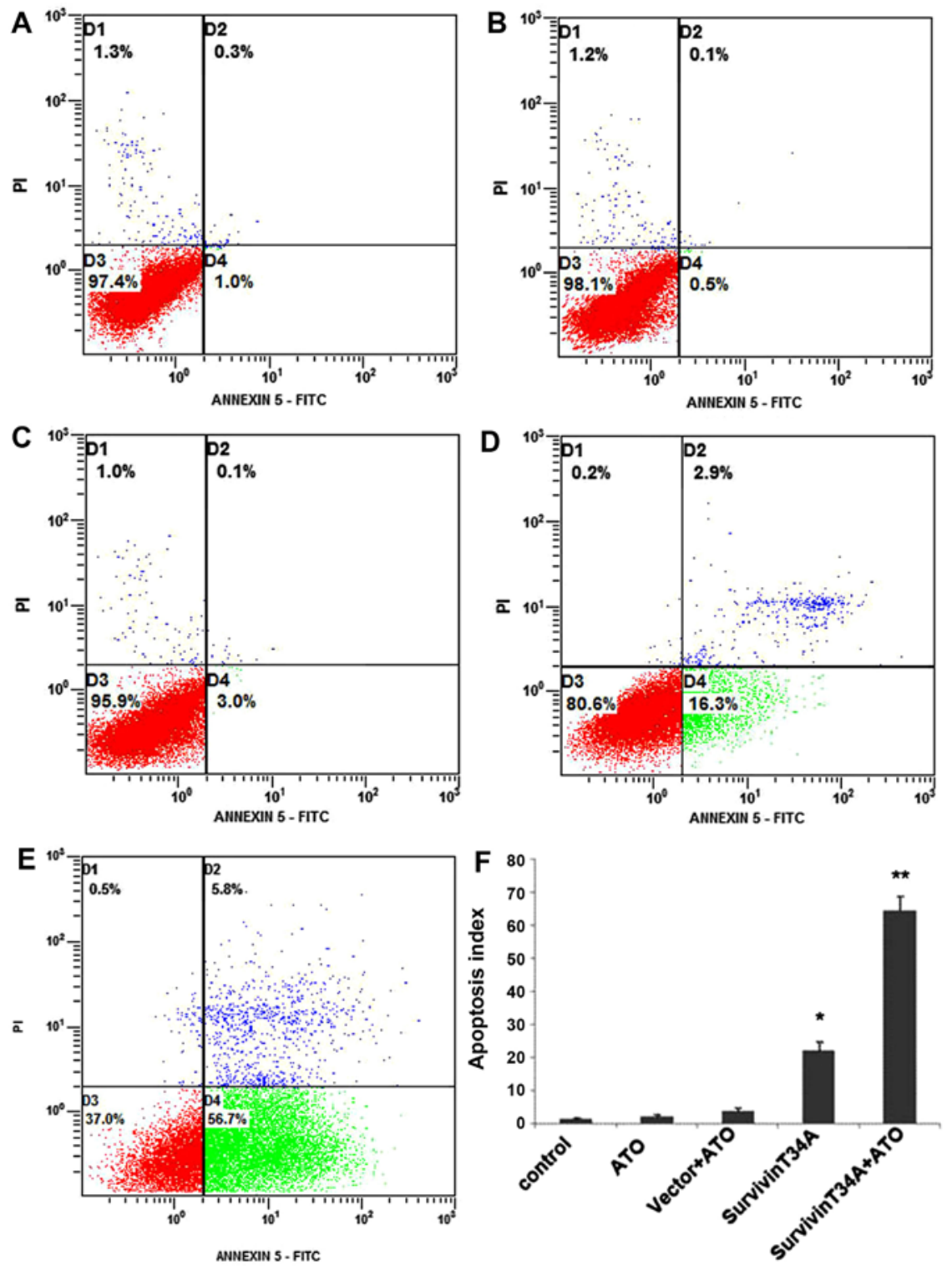

Figure 1. Cytotoxicity of the survivinT34A+arsenic trioxide combination on Hepa1-6 cells. The cells harvested were processed for apoptosis assessment by using the Annexin V-FITC/PI dual labeling technique. Hepal-6 cells were untreated (A) or treated with ATO (B), vector+ATO (C), survivinT34A (D), and combined therapy (E) for $12 \mathrm{~h}$, the average apoptosis of harvested Hepa1-6 cells (F), the data are expressed as percentages. ${ }^{*} \mathrm{P}<0.05,{ }^{\text {,** }} \mathrm{P}<0.01$; columns, mean; bars, SD.

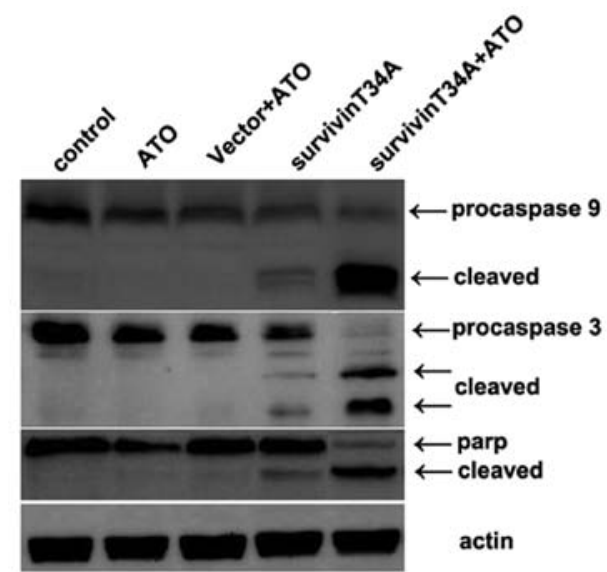

Figure 2. Effects of HBx on the expression of caspase-3, -9, PARP and their cleaved forms. Hepa1-6 cells were treated as mentioned above for $48 \mathrm{~h}$ and then we analyzed the expression levels of caspase-3, -9, PARP and their cleaved forms by western blotting. $\beta$-actin was used as a loading control. using the Rho123 fluorescence dye. As shown in Fig. 5, the combinational treatment results in significant reduction of Rho123 fluorescence compared to that observed in other treatment groups. In addition, this relocation was inhibited by $\mathrm{N}$-acetyl-1-cysteine (NAC). This indicates that the effect of survivinT34A in enhancing $\mathrm{As}_{2} \mathrm{O}_{3}$ induced-apoptosis involves mitochondrial damage.

Enhanced antitumor effect by combination treatment in vivo. On the basis of the in vitro pro-apoptotic effects of survivinT34A and ATO, we further examined the combined anti-neoplastic effect of survivinT34A and ATO on Hepa1-6 tumors in vivo. Tumor volume of mouse assay monitored every 3 days showed that the tumor growth was significantly inhibited by treatment with survivinT34A+ATO while either survivinT34A or ATO exhibits moderate antitumor efficiency. After 30 days, the average tumor volumes in mice treated with 

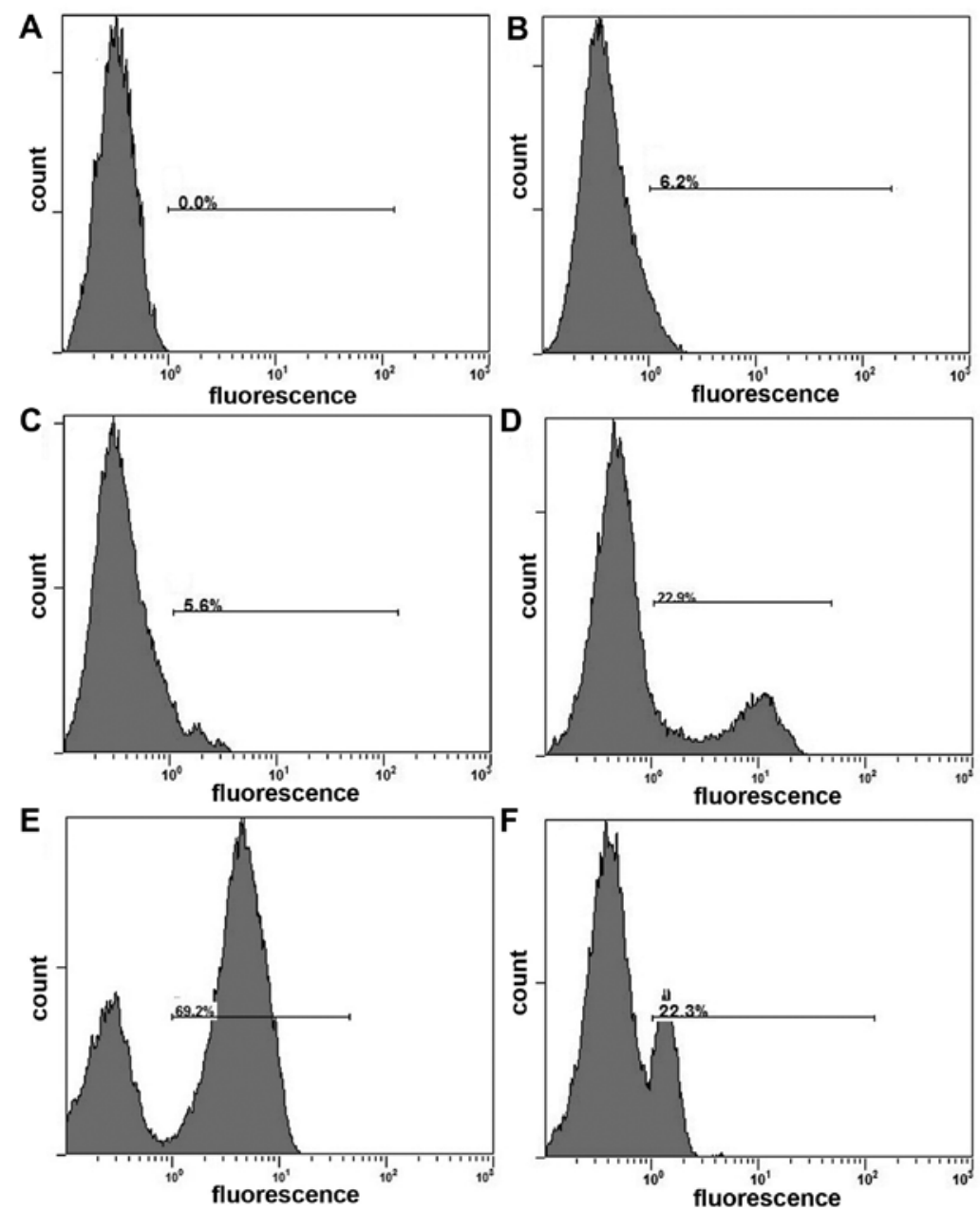

Figure 3. Detection of intracellular ROS in the effects of combined treatment with survivinT34A+arsenic trioxide in Hepa1-6 cells. Hepa1-6 cells were untreated (A) or treated with ATO (B), vector+ATO (C), survivinT34A (D), the two-drug combination (E), or the combination plus $2.5 \mathrm{mM} \mathrm{NAC}$ for $20 \mathrm{~h}$ (F), and then stained with DCFH-DA; fluorescence was measured by flow cytometry.
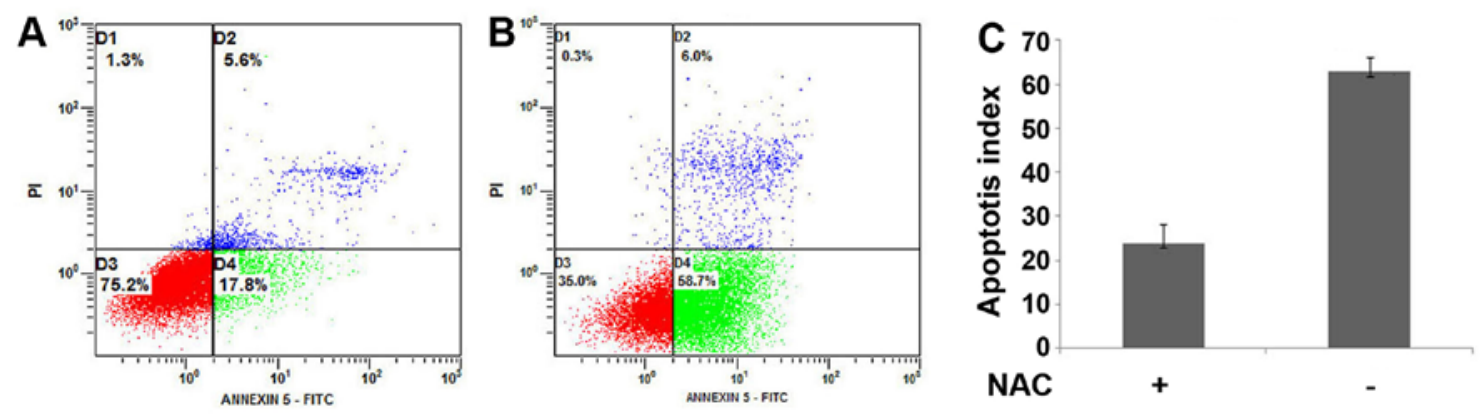

Figure 4. NAC markedly attenuated the cytotoxic effects induced by co-treatment with survivinT34A and $\mathrm{As}_{2} \mathrm{O}_{3}$. Hepa1-6 cells were pretreated with (A) or without (B) $2.5 \mathrm{mM} \mathrm{NAC}$ for $30 \mathrm{~min}$, followed by a 48-h treatment with combination treatment, and then were processed for apoptosis assessment by using the Annexin V-FITC/PI dual labeling technique. Representative results of three independent experiments and the average apoptosis of harvested Hepa1-6 cells (C) are shown, the data are expressed as percentages. " $\mathrm{P}<0.05$, columns, mean; bars, $\mathrm{SD}$.

NS, vector+ATO, ATO, survivinT34A, and combined therapy were $2074.56 \pm 474.532,2084.86 \pm 495.79,1901.03 \pm 463.81$, $1313.88 \pm 212.87$ and $573.82 \pm 121.29 \mathrm{~mm}^{3}$, respectively (Fig. 6). Similar results were also observed in the tumor weight. The average weight of tumors in the combination treatment group obviously declined compared with survivinT34A alone or other controls (data not shown). To validate whether the cell death came from apoptosis, apoptotic cells in tumor tissue were detected by TUNEL analysis. The results revealed that the apoptotic cells in combination treatment group increased significantly compared to any of the controls (Fig. 7). The apoptotic index in the tumor tissues from the combination treatment group was $40.6 \pm 5.8 \%$ when compared with all controls (Fig. 7). These results indicated that the co-treatment with survivinT34A and arsenic trioxide also enhanced cell apoptosis in Hepa1-6 tumors grafted in C57BL/6 mice. 

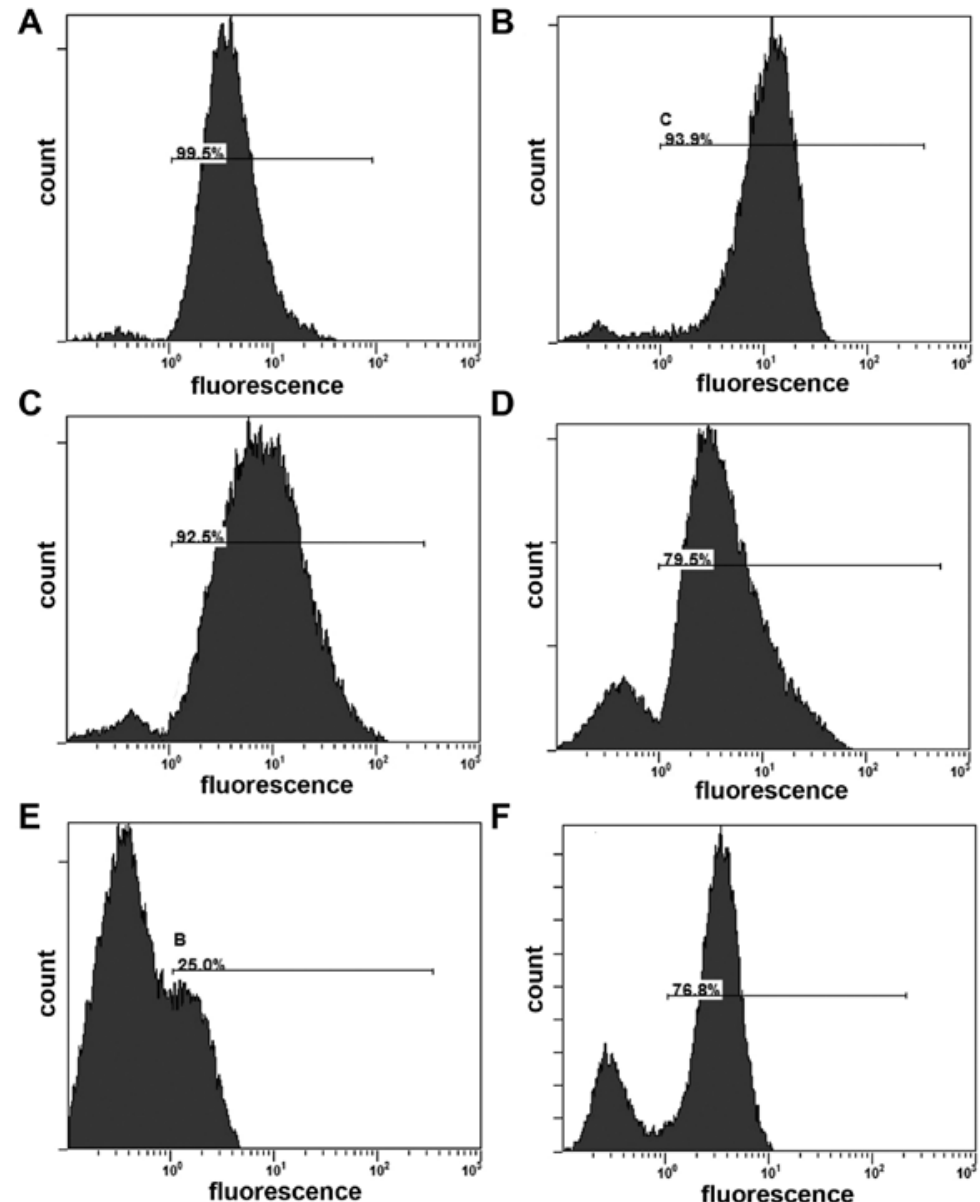

Figure 5. Assessment of mitochondrial transmembrane potential $\left(\Delta \Psi_{\mathrm{m}}\right)$ collapse in Hepa1-6 cells. After treatment as described in Fig. 2, Hepa1-6 cells were stained with the potential-sensitive dye Rho1 23 at $100 \mathrm{nM}$ for $15 \mathrm{~min}$ at $37^{\circ} \mathrm{C}$, and fluorescence was measured by flow cytometry. The precentage of cells with Rhodamine 123 fluorescence was $99.5 \%$ (A), 93.9\% (B), 92.5\% (C), 79.5\% (D) and 25\% (E) in each treatment group, respectively, implying that two-drug combination induced the loss of $\Delta \Psi_{\mathrm{m}}$. Pretreatment with $2.5 \mathrm{mM} \mathrm{NAC}$ inhibit the loss of $\Delta \Psi_{\mathrm{m}}$ caused by two-drug combination (F). The data represent the results of three experiments.

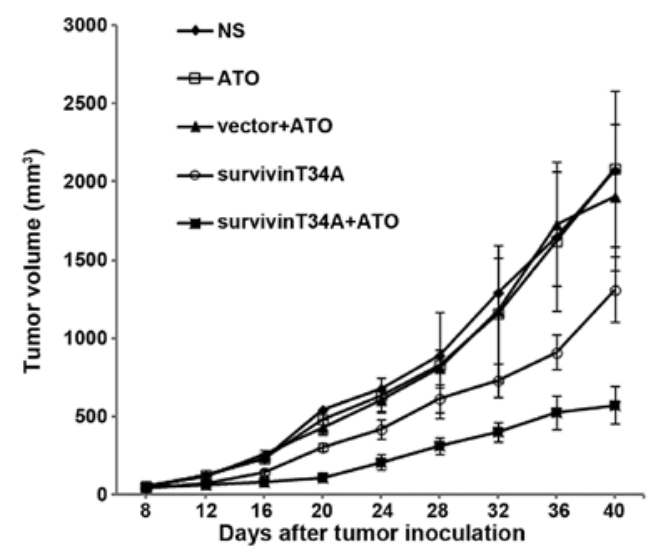

Figure 6. In vivo efficacy and specificity of survivinT34A+arsenic trioxide combination in hepatocellular carcinoma growth. Mice were i.v. administered with $5 \mu \mathrm{g}$ survivinT34A/15 $\mu \mathrm{g}$ liposome complexes+ATO; $5 \mu \mathrm{g}$ survivinT34A/15 $\mu \mathrm{g}$ liposome complexes; ATO (3 mg/kg); vector+ATO; $100 \mu 1$ of $0.9 \% \mathrm{NaCl}$ solution (NS), respectively every 2 days. Significant tumor regression of survivinT34A combined with arsenic trioxide treated mice was observed in the growth curve $\left({ }^{*} \mathrm{P}<0.001\right)$.

\section{Discussion}

It is well known that treatment with survivinT34A or ATO alone induces apoptosis via different mechanism. Phosphorylation at Thr34 of survivin is critical for its regulation by cyclindependent kinase, $\mathrm{p} 34 \mathrm{cdc}$-cyclin B1, which increased survivin expression. Moreover, the Thr34 phosphorylation site is responsible for stabilizing anti-apoptotic protein-protein interactions through the BIR domain of survivin (11). However, the mutant survivin of Thr34-Ala (T34A) cannot be phosphorylated by p34cdc2-cyclin B1, leading to the dissociation of a survivincaspase-9 complex and subsequent mitochondrial-dependent apoptosis with release of cytochrome $c$ and loss of mitochondrial transmembrane potential during cell division (13). Whereas, ATO biochemically binds to vicinal thiol groups of proteins with high affinity and biologically exerts its pleiotropic toxic effects via several types of reaction including modulation of the intracellular glutathione redox system and oxidative injury (28), induction of mitotic arrest (29), DNA damage due to the inhibition of DNA repair (30). As increasingly evident, therapies based on the combination of anticancer agents with non-overlapping mechanisms of action can result in enhanced anticancer efficacy and reduced adverse side-effects. The targeted disruption of survivin has been proved effective in improving the efficacy of chemotherapy- and/or radiotherapy-induced apoptosis in resistant tumor cells $(31,32)$. In this regard, we examined whether the survivinT34A, which is capable of directly inducing cell death, could enhance the efficacy of ATO. 

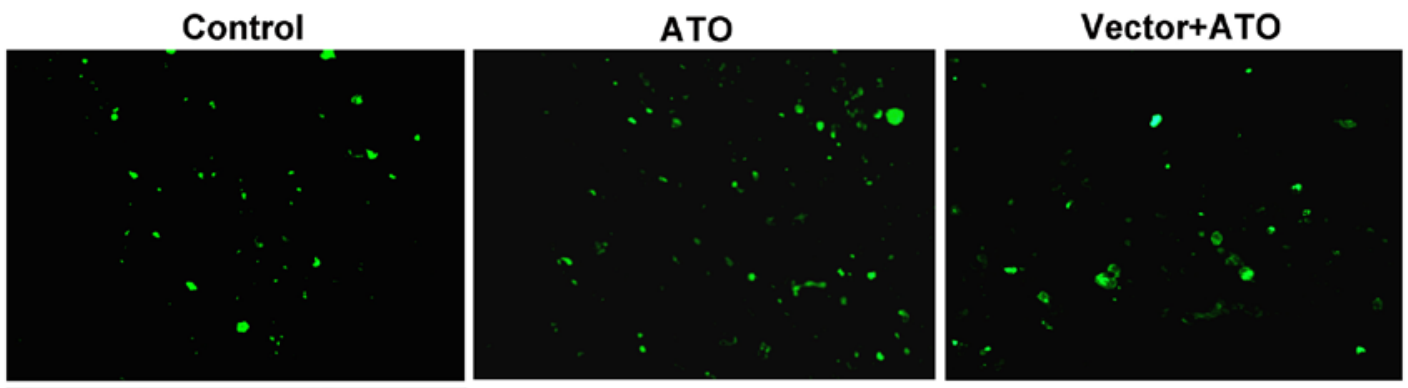

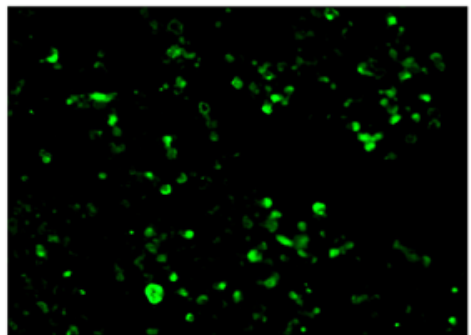

SurvivinT34A

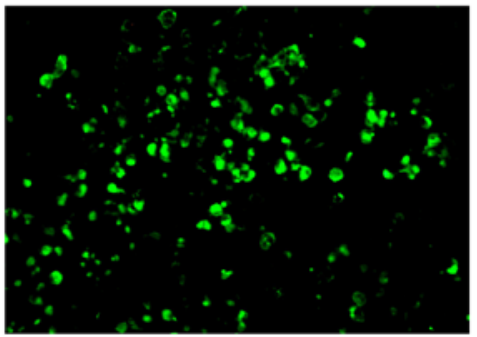

SurvivinT34A+ATO

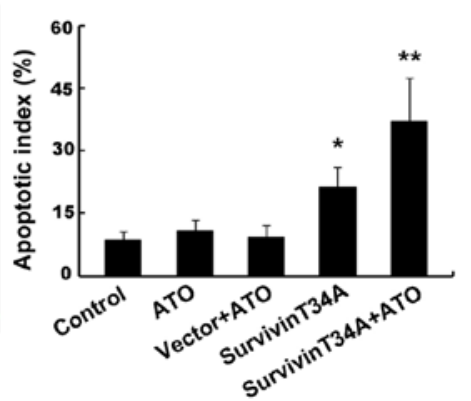

Figure 7. Apoptosis assays of tumor tissues in different groups. TUNEL staining of tumors revealed that survivinT34A/liposome complexes+ATO induced a significant enhancement of apoptotic cells in contrast to control therapies; magnification, $\mathrm{x} 400$. Apoptosis index of survivinT34A/liposome complexes+ATOtreated tumor was much higher than that of control groups.

In the present study, several observations were made concerning the mechanism of synergistic antitumor effect by treatment that combines survivinT34A with Arsenic trioxide. To the best of our knowledge, the present study has, for the first time, demonstrated that disruption of survivin by survivinT34A augments ATO-induced antitumor activity in Hepa1-6 both in vitro and in vivo. Two major implications can be obtained from the increased antitumor effects of survivinT34A and ATO. First, an interruption of survivin levels plays an important role in reducing apoptosis of HCC. In agreement with this, several studies have concluded that survivin expression was correlated with poor prognosis in patients with HCC, and upregulation of survivin is an independent risk factor in hepatocellular carcinoma cell survival as well as in resistance to apoptosis (33). Second, and most importantly, survivinT34A increases arsenic trioxide-induced apoptosis. This observation is supported by the present findings. The treatment that combines survivinT34A with ATO significantly increased the apoptosis of cells when compared with the survivinT34A or ATO alone. The level of cheavage of procaspase-3 and -9 into the active form remarkably increased in the combination group compared with the survivinT34A or ATO groups, suggesting that the initiation of apoptosis is through the intrinsic pathway and the process of apoptosis has bypassed the point-of-no-return in the apoptotic cascade. Thus, it is indicated that increased induction of apoptosis is crucial for survivinT34A-induced enhancement of the antitumor effects of ATO in HCC cells.

Cellular reactive oxygen species (ROS) are the major components of the endogenous oxidants, which is essential to cell survival, but the effect of ROS on cells is complex. At high level, oxidative stress may stimulate cells to undergo apoptosis, whereas in low concentration, it may initiate proliferative or survival signaling to antagonize apoptosis (34). This implies that two antagonizing signaling pathways coexist in the process in which cancer cells are induced to undergo apoptosis by cytotoxic drugs. Therefore, the effects of ROS depend on their levels. ROS accumulation has been demonstrated to play a pivotal role in the process in which ATO triggers tumor cell death (26). Our results suggest that the elevation of cellular ROS level elicited by survivinT34A plus ATO may surpass a certain threshold that finally overrides anti-apoptotic forces, shifting the cell survival/death balance towards cell death. NAC, as an antioxidant and ROS scavenger, could inhibit the two-drug combination treatment-induced apoptotic death. This strongly indicates that the synergistic efficacy that survivinT34A and arsenic trioxide exert on Hepa1-6 cancer cells is ROS dependent. It has been proven that excessive ROS production may result in reduction of the mitochondrial membrane potential and impair the mitochondrial respiratory chain (27). In the present study, we also suggest that the elevating intracellular ROS level induced by the survivinT34A- $\mathrm{As}_{2} \mathrm{O}_{3}$ combined therapy is essential for the loss of mitochondrial membrane potential.

In our laboratory, Peng et al (35) utilized survivinT34A plasmid ( $25 \mu \mathrm{g} / \mathrm{one})$ complexed with cationic liposome (DOTAP:chol) by intravenous administration to inhibit tumor growth in female BALB/c mice bearing subcutaneous 4T1 mammary carcinoma. In another study by Li et al (36) investigated the synergistic antitumor effect by combining liposome-encapsulated mouse survivinT34A (20 $\mu \mathrm{g} / \mathrm{one})$ and hyperthermia in mouse colon cancer models. In this study, the gene delivery system we utilized was the same as previously reported $(35,36)$, which proved to efficiently increase the therapeutic efficacy of the survivinT34A plasmid. Our previous biodistribution studies have showed that cationic lipid-DNA complexes was accumulated mainly in tumor tissues (37). This is due to the leaky microvasculature in tumors that could facilitate extravasation, and thus, increase the permeability of tumor vessels to liposomes.

In summary, the present study showed that the combination therapy of survivinT34A and arsenic trioxide exhibited 
synergistic induction of apoptosis in hepatocellular carcinoma cells both in vitro and in vivo, and this synergy might result from elevation of intracellular ROS level and mitochondrial damage. Therefore, this study particularly indicated not only a rational basis for combination therapy with survivin suppression plus arsenic trioxide, but also a novel therapeutic regimens for the treatment of some cancers that show failure to chemotherapy and/or radiotherapy in clinical setting.

\section{Acknowledgements}

This study was supported by the Science and Technology Support Program of Sichuan (2014SZ0122) and the National Natural Science Foundation of China (grant no. 81101728).

\section{References}

1. Altekruse SF, McGlynn KA and Reichman ME: Hepatocellular carcinoma incidence, mortality, and survival trends in the United States from 1975 to 2005. J Clin Oncol 27: 1485-1491, 2009.

2. Altieri DC: The molecular basis and potential role of survivin in cancer diagnosis and therapy. Trends Mol Med 7: 542-547, 2001

3. Gąsowska-Bodnar A, Bodnar L, Dąbek A, Cichowicz M, Jerzak M, Cierniak S, Kozłowski W and Baranowski W: Survivin expression as a prognostic factor in patients with epithelial ovarian cancer or primary peritoneal cancer treated with neoadjuvant chemotherapy. Int J Gynecol Cancer 24: 687-696, 2014.

4. Liu JL, Gao W, Kang QM, Zhang XJ and Yang SG: Prognostic value of survivin in patients with gastric cancer: A systematic review with meta-analysis. PLoS One 8: e71930, 2013.

5. Deveraux QL and Reed JC: IAP family proteins - suppressors of apoptosis. Genes Dev 13: 239-252, 1999.

6. Kanwar JR, Shen WP, Kanwar RK, Berg RW and Krissansen GW: Effects of survivin antagonists on growth of established tumors and B7-1 immunogene therapy. J Natl Cancer Inst 93: 1541-1552, 2001.

7. Pennati M, Colella G, Folini M, Citti L, Daidone MG and Zaffaroni N: Ribozyme-mediated attenuation of survivin expression sensitizes human melanoma cells to cisplatin-induced apoptosis. J Clin Invest 109: 285-286, 2002.

8. Jiang G, Li J, Zeng Z and Xian L: Lentivirus-mediated gene therapy by suppressing survivin in BALB/c nude mice bearing oral squamous cell carcinoma. Cancer Biol Ther 5: 435-440, 2006.

9. Pisarev V, Yu B, Salup R, Sherman S, Altieri DC and Gabrilovich DI: Full-length dominant-negative survivin for cancer immunotherapy. Clin Cancer Res 9: 6523-6533, 2003.

10. Grossman D, Kim PJ, Schechner JS and Altieri DC: Inhibition of melanoma tumor growth in vivo by survivin targeting. Proc Natl Acad Sci USA 98: 635-640, 2001.

11. Verdecia MA, Huang H, Dutil E, Kaiser DA, Hunter T and Noel JP: Structure of the human anti-apoptotic protein survivin reveals a dimeric arrangement. Nat Struct Biol 7: 602-608, 2000.

12. Muchmore SW, Chen J, Jakob C, Zakula D, Matayoshi ED, Wu W, Zhang H, Li F, Ng SC and Altieri DC: Crystal structure and mutagenic analysis of the inhibitor-of-apoptosis protein survivin. Mol Cell 6: 173-182, 2000.

13. O'Connor DS, Grossman D, Plescia J, Li F, Zhang H, Villa A, Tognin S, Marchisio PC and Altieri DC: Regulation of apoptosis at cell division by $\mathrm{p} 34 \mathrm{cdc} 2$ phosphorylation of survivin. Proc Natl Acad Sci USA 97: 13103-13107, 2000.

14. Sanz MA, Grimwade D, Tallman MS, Lowenberg B, Fenaux P, Estey EH, Naoe T, Lengfelder E, Büchner T, Döhner H, et al: Management of acute promyelocy tic leukemia: Recommendations from an expert panel on behalf of the European LeukemiaNet. Blood 113: 1875-1891, 2009.

15. Momeny M, Zakidizaji M, Ghasemi R, Dehpour AR, RahimiBalaei M, Abdolazimi Y, Ghavamzadeh A, Alimoghaddam K and Ghaffari SH: Arsenic trioxide induces apoptosis in NB-4, an acute promyelocytic leukemia cell line, through up-regulation of p73 via suppression of nuclear factor kappa B-mediated inhibition of p73 transcription and prevention of NF-kappaB-mediated induction of XIAP, cIAP2, BCL-XL and survivin. Med Oncol 27: 833-842, 2010
16. Ghaffari SH, Bashash D, Dizaji MZ, Ghavamzadeh A and Alimoghaddam K: Alteration in miRNA gene expression pattern in acute promyelocytic leukemia cell induced by arsenic trioxide: a possible mechanism to explain arsenic multi-target action. Tumour Biol 33: 157-172, 2012

17. Gazitt Y and Akay C: Arsenic trioxide: An anticancer missile with multiple warheads. Hematology 10: 205-213, 2005.

18. Seol JG, Park WH, Kim ES, Jung CW, Hyun JM, Kim BK and Lee YY: Effect of arsenic trioxide on cell cycle arrest in head and neck cancer cell line PCI-1. Biochem Biophys Res Commun 265: 400-404, 1999.

19. Dizaji MZ, Malehmir M, Ghavamzadeh A, Alimoghaddam K and Ghaffari SH: Synergistic effects of arsenic trioxide and silibinin on apoptosis and invasion in human glioblastoma U87MG cell line. Neurochem Res 37: 370-380, 2012.

20. Lin CC, Hsu C, Hsu CH, Hsu WL, Cheng AL and Yang CH: Arsenic trioxide in patients with hepatocellular carcinoma: A phase II trial. Invest New Drugs 25: 77-84, 2007.

21. Vuky J, Yu R, Schwartz L and Motzer RJ: Phase II trial of arsenic trioxide in patients with metastatic renal cell carcinoma. Invest New Drugs 20: 327-330, 2002.

22. Kim KB, Bedikian AY, Camacho LH, Papadopoulos NE and McCullough C: A phase II trial of arsenic trioxide in patients with metastatic melanoma. Cancer 104: 1687-1692, 2005.

23. Kumar P, Gao Q, Ning Y, Wang Z, Krebsbach PH and Polverini PJ: Arsenic trioxide enhances the therapeutic efficacy of radiation treatment of oral squamous carcinoma while protecting bone. Mol Cancer Ther 7: 2060-2069, 2008.

24. Wu YC, Yen WY, Lee TC and Yih LH: Heat shock protein inhibitors, 17-DMAG and KNK437, enhance arsenic trioxide-induced mitotic apoptosis. Toxicol Appl Pharmacol 236: 231-238, 2009.

25. Zhang N, Wu ZM, McGowan E, Shi J, Hong ZB, Ding CW, Xia P and Di W: Arsenic trioxide and cisplatin synergism increase cytotoxicity in human ovarian cancer cells: Therapeutic potential for ovarian cancer. Cancer Sci 100: 2459-2464, 2009.

26. Li JX, Shen YQ, Cai BZ, Zhao J, Bai X, Lu YJ and Li XQ: Arsenic trioxide induces the apoptosis in vascular smooth muscle cells via increasing intracellular calcium and ROS formation. Mol Biol Rep 37: 1569-1576, 2010.

27. Park MT, Kang YH, Park IC, Kim CH, Lee YS, Chung HY and Lee SJ: Combination treatment with arsenic trioxide and phytosphingosine enhances apoptotic cell death in arsenic trioxide-resistant cancer cells. Mol Cancer Ther 6: 82-92, 2007.

28. Jing Y, Dai J, Chalmers-Redman RM, Tatton WG and Waxman S: Arsenic trioxide selectively induces acute promyelocytic leukemia cell apoptosis via a hydrogen peroxide-dependent pathway. Blood 94: 2102-2111, 1999.

29. Li YM and Broome JD: Arsenic targets tubulins to induce apoptosis in myeloid leukemia cells. Cancer Res 59: 776-780, 1999.

30. Yoo DR, Chong SA and Nam MJ: Proteome profiling of arsenic trioxide-treated human hepatic cancer cells. Cancer Genomics Proteomics 6: 269-274, 2009.

31. Tirrò E, Consoli ML, Massimino M, Manzella L, Frasca F, Sciacca L, Vicari L, Stassi G, Messina L, Messina A, et al: Altered expression of c-IAP1, survivin, and Smac contributes to chemotherapy resistance in thyroid cancer cells. Cancer Res 66: 4263-4272, 2006.

32. Konduri S, Colon J, Baker CH, Safe S, Abbruzzese JL, Abudayyeh A, Basha MR and Abdelrahim M: Tolfenamic acid enhances pancreatic cancer cell and tumor response to radiation therapy by inhibiting survivin protein expression. Mol Cancer Ther 8: 533-542, 2009.

33. Liu JL, Zhang XJ, Zhang Z, Zhang AH, Wang W and Dong JH: Meta-analysis: Prognostic value of survivin in patients with hepatocellular carcinoma. PLoS One 8: e83350, 2013.

34. Burdon RH: Control of cell proliferation by reactive oxygen species. Biochem Soc Trans 24: 1028-1032, 1996.

35. Peng XC, Yang L, Yang LP, Mao YQ, Yang HS, Liu JY, Zhang DM, Chen LJ and Wei YQ: Efficient inhibition of murine breast cancer growth and metastasis by gene transferred mouse survivin Thr34 $\rightarrow$ Ala mutant. J Exp Clin Cancer Res 27: 46, 2008.

36. Li ZM, Zhao YW, Zhao CJ, Zhang XP, Chen LJ, Wei YQ and Yang HS: Hyperthermia increases the therapeutic efficacy of survivinT34A in mouse tumor models. Cancer Biol Ther 12: 523-530, 2011.

37. Huang AL, Wan Y, Liao DY, Hu HZ, Wei L, Wang XH, Wen YJ, Li J, Chen LJ, Kan B, et al: Suppression of human MDA-MB-435S tumor by U6 promoter-driven short hairpin RNAs targeting focal adhesion kinase. J Cancer Res Clin Oncol 136: 1229-1242, 2010. 